\title{
Formation of Negative lons on the Surface of a Solid Body and Their Influence on the Thermoelectronic and Autoelectronic Emission of Free Electrons
}

\author{
Leonid Ivanovich Gretchikhin \\ Department of Information and Communication Technologies, Belarusian State Academy of Communications, Minsk, Belarus
}

Email address:

Gretchihin@yandex.ru

To cite this article:

Leonid Ivanovich Gretchikhin. Formation of Negative Ions on the Surface of a Solid Body and Their Influence on the Thermoelectronic and Autoelectronic Emission of Free Electrons. American Journal of Applied Scientific Research. Vol. 5, No. 3, 2019, pp. 47-55.

doi: 10.11648/j.ajasr.20190503.11

Received: August 29, 2019; Accepted: September 19, 2019; Published: October 14, 2019

\begin{abstract}
On the surface of the main crystal, positive ions interact with a negative cloud of free electrons and turn into neutral atoms. Neutral atoms having affinity to the electron other than zero additionally capture the electron and become negative ions. It is shown that under the action of ponderomotive forces the crystal surface is noticeably deformed, and it is experimentally confirmed. Besides, it is experimentally proved that three-atomic molecules possess allotropy in distribution of the built-in electric moments. The analysis of different physical models, which describe the thermo-and auto-electric emission of free electrons, is carried out and their inconsistency is shown. Thermal electron and field emission arise due to the temperature population of negative ions on the crystal surface and cluster vibrations in the near-surface layer. Field emission is thermal electron emission from negative ions and the Fermi level, which is transformed due to the action of the external electric field on the work of the surface by ponderomotive forces.
\end{abstract}

Keywords: Thermal Electron Emission, Field Emission, Negative Ions, Work Function, Electron Affinity Energy, Surface

\section{Introduction}

In the modern conditions of science and technology development, interest in negative ions has increased significantly. In this connection, it has been established in [1] that negative ions are formed as a result of the interaction of a free electron with the built-in and induced electrical moment of atoms, molecules and cluster formations. This results in a static, rather than dynamic, system of interacting electric charges. The formation of negative ions is realized in different conditions: 1-when adsorption and spraying of atoms, molecules or clusters on the surface of a solid body surface, which have a noticeable affinity to the electron; 2-in cathode spots of electric discharges and in low-temperature plasma of the arc and spark discharge channel, as well as under the influence of powerful radiant fluxes produced by hot gases and laser radiation; 3-directly on the surface of a solid body of atoms, molecules, clusters of the basic material.

In the process of physical or chemical adsorption or by sputtering on the surface of a solid body, a certain proportion of particles is in the form of negative ions. Thus, the physical adsorption of oxygen atoms takes place on artificial Earth satellites flying at high altitudes in the free molecular flow regime. This leads to the electrification of satellites and the appearance of a powerful non-equilibrium violet blue glow in the frontal hemisphere and red glow in the rear area of the satellite flight (Gretchikhin effect) [2]. Spraying of heterogeneous atoms or molecules with electron affinity leads to the appearance of $\mathrm{p}-, \mathrm{n}$-conductivity and $\mathrm{p}-\mathrm{n}$ transition [4].

In electric discharges, powerful negative ion fluxes flow out of the cathode slicks, which leads to the occurrence of reverse electric currents directly near the cathode slick, which is an order of magnitude and more superior to the main discharge current. This leads to the fact that the torch of the cathode spot is deflected in the magnetic field not according to the Ampere law [6]. The temperature of arc and spark discharge plasma electrons is determined by the process of ionization of negative ions [6].

The effect of powerful radiant fluxes formed by strongly 
heated gases or lasers on the surface of a solid body does not lead to a constant continuous destruction of the surface of a solid body, but to an explosive mechanism in the form of separate emissions of plasma torches. This made it possible to carry out the flights of rockets and satellites in the Earth's atmosphere with the second space velocity, as in this case the destruction of the heat protection coating occurs with a lower speed than at the first space velocity [2].

Directly on the surface of a solid body, the atoms of the main material are also in the form of negative ions. This area is not sufficiently developed and requires detailed research. As a result, a specific goal has arisen: to create an adequate physical model of the formation of negative ions of the basic material and to justify the conditions when negative ions predominantly determine the physics of the phenomenon. And it primarily concerns the emission properties of the crystal surfaces. In order to achieve this goal, it is necessary to solve the following problems:

1. Clearly substantiate the structure of crystals.

2. Determine the probability of formation of negative ions on the surface of the crystals.

3. Find out what influence the external electric field has on the deformation of the crystal surface.

4. Substantiate the model of electronic emission from the crystal surface.

5. Perform experimental studies.

Let us successively consider all mentioned tasks.

\section{Formation of Crystals}

Depending on the temperature, each substance resides in different states of aggregation. Starting from high temperatures, the substance remains in the plasma state, and then, sequentially, with the reduction of temperature, it passes into the gaseous, liquid, solid (crystalline) and densely packed state $[7,8]$. Each state is formed by its own particles. Plasma contains both positive and negative ions, as well as electrons. The gaseous state contains atoms and molecules. The solid state is formed by clusters that are composed of molecules. In the close-packed state, clusters are leveled and atoms create a single monolithic structure. The boundary of transition from one state into another one is quite blurred. This is due to emergence of different types of interactions and relationships between atoms, molecules and clusters.

Interaction between atoms causes formation of diatomic molecules, an atom with a diatomic molecule forms a triatomic molecule. Diatomic or triatomic molecules form cluster structures. By interacting with each other, cluster structures form a crystal lattice. Separate neutral atoms are present in inter-cluster voids. Up to the melting temperature, one atom is present in the inter-cluster void, and at the melting temperature, two atoms are converted into a diatomic molecule [7, 8]. Formation of diatomic molecules in inter-cluster voids causes an increase in the specific resistance of metals by exactly two times, which was experimentally proved [9].

The first, then the second, and subsequently the third coordination layer is formed around the central atom in the course of formation of cluster structures. Atoms of the third coordination layer combine clusters into a single monolithic structure, i.e., closely packed [19]. Clusters dissolve with that. See the experimental proof of this phenomenon in [10]. The close-packed state possesses superconductivity [11]. The monocluster layer at the surface of the crystal is the layer with its own typical features for each substance. When atoms interact, valence electrons are exchanged and, as a result of this, they generalize and form an electron cloud, which is described by the Fermi-Dirac distribution law. The electron energy distribution was obtained from the X-ray spectra for a number of metals inside the crystal and above the surface, and the corresponding Fermi levels were determined. The theoretical calculation was developed by the author and described in [7]. The calculations were performed for crystals of silicon, germanium, aluminum, iron, copper, nickel, boron, titanium, sodium, lithium, beryllium. As a result, values of the Fermi levels, displacements of the ionization boundary, and values of the forbidden bands were obtained. The comparison with the experiment was performed for a crystal of aluminum. See the result in Figure 1 [7], and see the result for a semiconductor silicon crystal in Figure 2 [7].

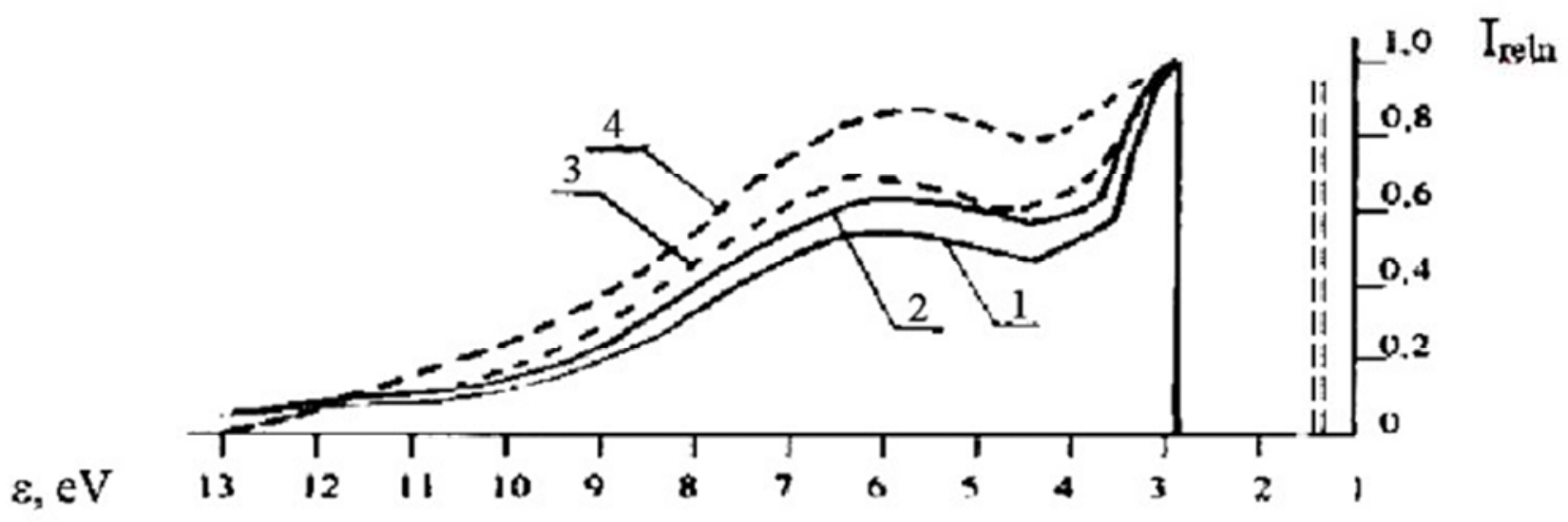

Figure 1. Distribution of electrons by energy values for a crystal of aluminum: 1 - theoretical calculation; 2 - theoretical calculation with consideration of $U$-shaped hardware broadening with the energy of $0.05 \mathrm{eV} ; 3$-experimental distribution from the L3 band and 4 - from the $\mathrm{K}$ band. 


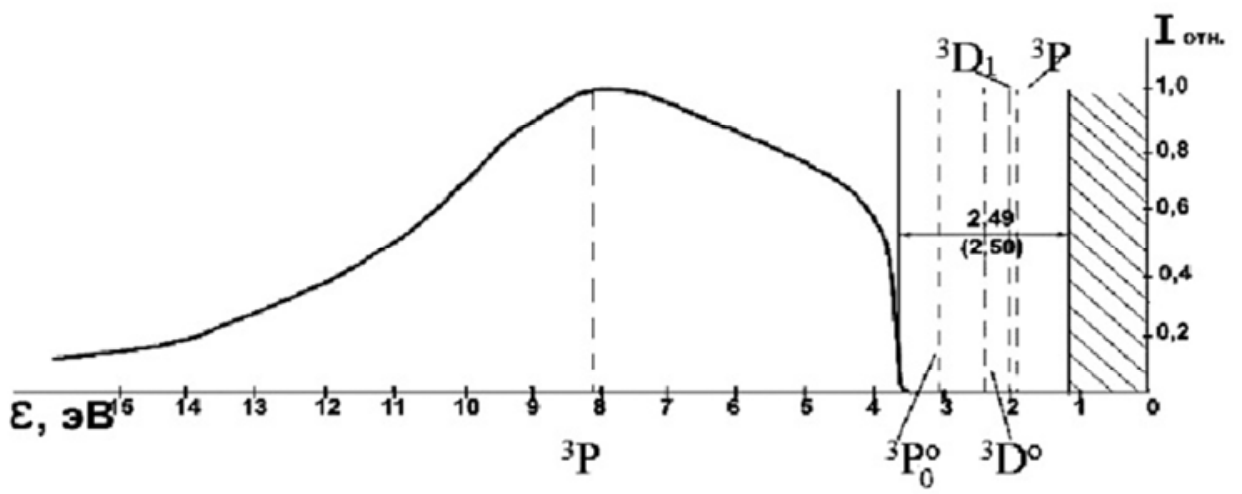

Figure 2. General view of the Fermi-Dirac distribution for a silicon crystal.

The silicon atom has the following configuration of electrons: $1 s^{2} 2 s^{2} 2 p^{6} 3 s^{2} 3 p^{2}$. The main state $3 p^{2}$ is even triplet $3 p^{2} P_{0}, 3 p^{23} P_{1}, 3 p^{23} P_{2}$. These energy levels can be combined with odd themes $4 s\left({ }^{3} P^{o}{ }_{0}\right), 4 s\left(P^{o}{ }_{1}\right), 4 s\left(P^{o}{ }_{2}\right) ; 3 s 3 p^{3}\left({ }^{\beta} D^{o}{ }_{1}\right)$, $3 s 3 p^{3}\left({ }^{3} D^{o}{ }_{2}\right), 3 s 3 p^{3}\left({ }^{3} D^{o}{ }_{3}\right)$; and through these terms with a group of triplet terms with excitation of the $4 p$ electron. As a result of this interaction, the main level of silicon has the broadening of $5.894 \mathrm{eV}$; the level of excitation of ${ }^{3} P^{o}{ }_{0,1,2}$ is $3.914 \mathrm{eV}$ with the probability of participation in formation of the valence band of $0.212 ;{ }^{3} D^{o}{ }_{1,2,3}$ is $2.829 \mathrm{eV}$ with the probability of participation in formation of the valence band of 0.157 ; group of terms $4 p\left(3 D_{1,2,3}\right)$ is $0.741 \mathrm{eV}$ with the probability of participation in formation of the valence band of 0.055 , and $4 p\left(P_{0,1,2}\right)$ is $0,511 \mathrm{eV}$ with the probability of 0.0304 . A similar analysis was performed for all the elements listed above.

Therefore, clusters are located in the surface layer of the crystal, over which a cloud of electrons resides, and a double electric layer is formed. Since the cluster lattice structure is positively charged, the positive charge of each cluster is neutralized by the corresponding charge of electrons, i.e., there is its own electron in the cloud above each atom of the cluster on the surface.

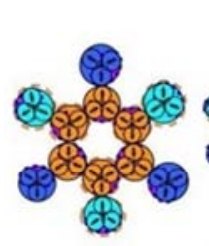

(a)

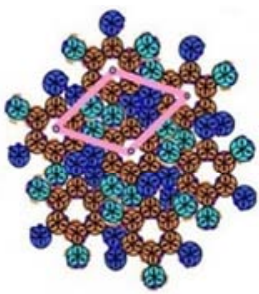

(b)

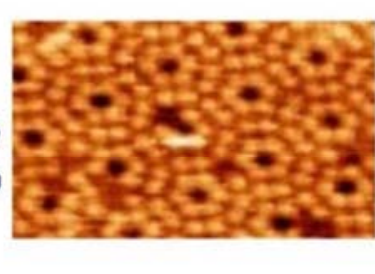

(c)
Figure 3. a) silicon cluster (theoretic); b) silicon Si surface (111) (theoretic); c) silicon emission surface (experiment).

The crystal oscillates with the increase in temperature. Crystal oscillations are caused by intercluster interaction. This approach is very fundamental. The densest packing is implemented for the (111) plane of body-centered crystalline structures. In this case, the packing corresponds to the intra-cluster one. Silicon surfaces were studied with theoretical justification for cluster structures using a scanning tunneling microscope (STM). See the theoretically calculated cluster type and the general appearance of the surface, as well as the experimental result obtained for the (111) silicon (Si) surface in Figure 3 [12].

It follows from this that silicon clusters are flat and must oscillate mainly perpendicularly to the surface with the increase in temperature. The obtained emission picture of the molecules that make up the cluster is quite blurry, which indicates that electron emission occurs from each individual atom in the molecule. When the cantilever (or the needle, the probe) of the STM is stopped at the maximum of the emission current, this occurs from a single atom.

To describe vibrations of cluster structures, not quantum statistics, but the Maxwell-Boltzmann statistics should be used. Inter-cluster interaction determines the melting temperature. The melting temperature of a silicon crystal is $1688 \mathrm{~K}$ [13], which corresponds to binding energy $3 k_{B} T / e=0.436$ at the distance between clusters based on Figure $3 \mathrm{c}$ of $r_{e}=2.8 \mathrm{~nm}$.

The Lennard-Jones potential can be used to describe the interaction between clusters. See the interaction potential for silicon clusters in Figure 4. The arrow in Figure 4 shows a fragment of vibration of a silicon cluster on a crystal surface in normal conditions.

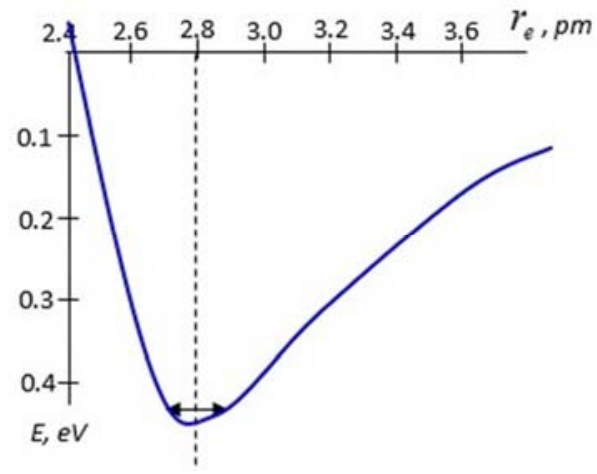

Figure 4. The cluster interaction potential normal to the surface.

As the cluster moves deeper into the crystal, the distance between the electron located on the Fermi surface is increased by the $\Delta r$ value and the interaction between the positive atomic ion in the cluster and the electron in the cloud is reduced. The difference between these energies is spent on excitation of valence electrons in the cloud. At normal 
temperature, the silicon cluster on the crystal surface oscillates with the average amplitude of $\Delta r=0.33 \AA$.

Each atom inside the crystal is a single ion, and they are held on the surface by the ion due to the random motion of electrons inside the crystal and exit to the surface and the atom is neutral. If such atom has electron affinity, then it turns into a negative ion.

\section{Negative Ions on the Crystal Surface}

The neutral atom on the crystal surface due to the electron-dipole interaction is in the form of a negative ion, as it is shown in $[12,14]$. See the energy diagram of the energy levels of negative silicon and indium ions relative to the bottom of the conduction band in Figure 5.

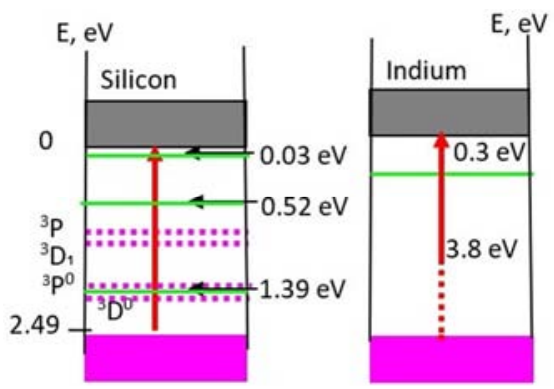

Figure 5. Energy levels of negative ions in the restricted band: a) of silicon and b) of indium.

A paradoxical situation arises: the crystal lattice inside the crystal consists of single positive ions, and they turn into negative ions on the surface.

If impurities are introduced into the main crystal, they are located in the interstices of the cluster lattice structure in the form of neutral atoms or molecules, and on the surface they turn into negative ions. Electrical, optical, and other properties of crystals are primarily determined by the surface layer. Therefore, the presence of negative ions on the surface of the crystal makes a significant contribution to various properties of crystals, and in some cases they are decisive. Let us consider the role of negative ions in emission properties of a crystal and, in particular, on thermal electron and field emission from these positions.

Thermal electron emission occurs from the Fermi level, which is substantially deformed due to vibration of clusters on the crystal surface. Field emission arises as a result of thermal electron emission and the effect of the external electric field on the crystal.

\section{Deformation of the Crystal Surface Upon Application of the External Electric Field}

The crystal structure is formed by cluster formations [7]. The size of cluster formations does not exceed $10 \mathrm{~nm}$, and the size of individual molecules is $\sim 1 \mathrm{~nm}$ [8]. At the voltage of $1 \mathrm{~V}$ on the STM cantilever, electric field strength of $E_{\ni} \approx 10^{8}$ $\mathrm{V} / \mathrm{m}$ will arise near the cluster surface, and the electric field strength of $E_{\ni} \approx 10^{9} \mathrm{~V} / \mathrm{m}$ for an individual atom will arise. With such electric field strengths, ponderomotive forces will arise, which will create pressure by acting on a single cluster, molecule or atom [15].

$$
\vec{P}=\sigma \vec{E}_{\ni}-\frac{\varepsilon_{0} \varepsilon_{r} E_{\ni}^{2}}{2},
$$

where $\sigma$ is the surface density of charges.

The first member $\sigma E_{\ni}$ in formula (1) determines the force of action of a negative ion on the electron along the electric field strength normal to the surface, and the second term causes arising of pressure along the crystal surface. See this situation demonstrated in Figure 6.

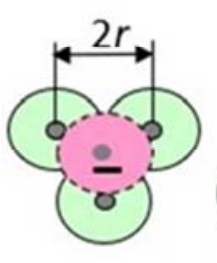

(a)

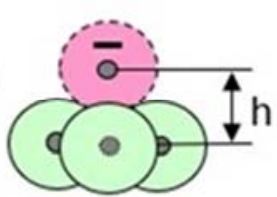

(b)

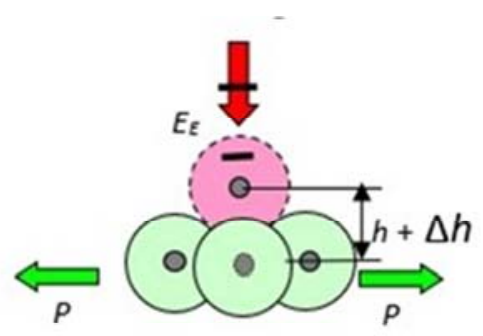

(c)

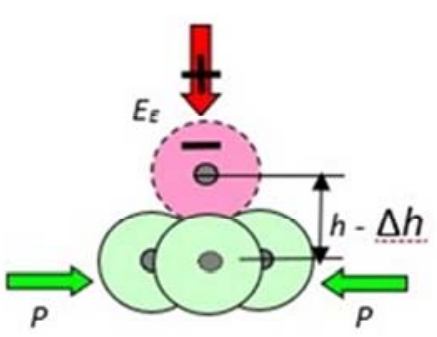

(d)

Figure 6. Location of molecules in the cluster: $a$-top view; $b$-side view; $c$-stretching with ponderomotive forces; $d$-compression with ponderomotive forces.

Tensile or compressive stress is determined by the energy density of the electric field and causes the corresponding deformation along the crystal surface.

$$
P=0.5 \varepsilon_{r} \varepsilon_{0} E_{\ni}^{2}=\frac{E \Delta l}{h},
$$

where $E$ is the Young's modulus.

In conditions of dynamic equilibrium

$$
\Delta l=\frac{P h}{2 E}, \text { a } h \cong \sqrt{3} \Delta l .
$$

Then the work of ponderomotive forces for stretching or 
compression is equal to

$$
\Delta W=\sqrt{3} \frac{\varepsilon_{0} \varepsilon_{r} E_{\ni}^{2} h}{2 E} r_{i}^{2}=\alpha E_{\ni}^{2},
$$

where $r_{i}$ is the radius of the negative ion, which should be considered as the radius of the neutral atom.

With the increase in the external electric field, negative ions on the crystal surface will automatically move somehow away from the STM cantilever deeper into the crystal, and vice versa, when compressed, they will approach the cantilever due to the extension of the surface layer, which will cause a reduction in the emission current in the first case and an increase in the emission current in the second case.

Linear resolution up to $1 \AA$ was reached in the STM. This means that when the cantilever moves along the molecule, emission current from each atom is recorded. See the deformation of the crystal surface in Figure 7.
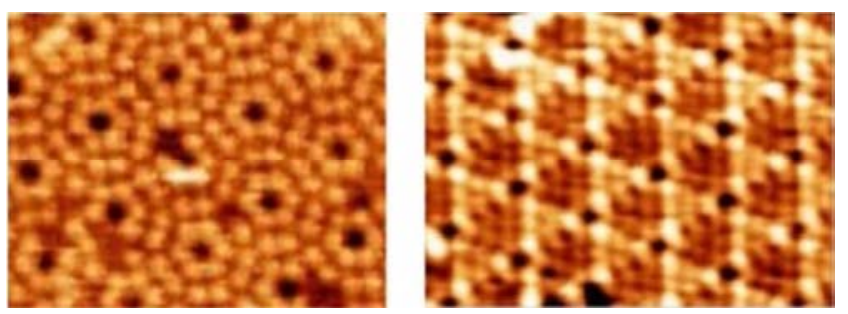

Figure 7. STM images of the Si (111) surface at various voltages on the probe: a) $\mathrm{Vs}=-2.0 \mathrm{~V}$; b) $\mathrm{Vs}=+2.0 \mathrm{~V}$.

In accordance with the model shown in Figure 5, we get an amazing coincidence. In additionally turns out that triatomic molecules have different allotropy, which was predicted in [12]. Only certain molecules are squeezed out in a strict order. With consideration of such deformation, field emission from individual atoms in a molecule at the negative voltage is less intense than the one at the positive voltage on the cantilever. To understand this, it is necessary to consider field emission from individual atoms on the surface from the standpoint of modern ideas about the structure and formation of negative ions on the crystal surface.

\section{Electron Emission from the Surface of Crystals}

\subsection{Models of Electronic Emission from the Surface of Crystals}

Based on the classical electronic theory of metals, Richardson obtained the formula for the saturation current density:

$$
J_{e}=C T^{1 / 2} \exp \left(-A_{B} / k_{b} T\right)
$$

Based on thermodynamic considerations and quantum statistics, Dashman received a slightly different formula

$$
J_{e}=A_{R} T^{2} \exp \left(-A_{B} / k_{b} T\right)
$$

where $A_{R}$ and $A_{B}$ were determined experimentally.

First serious experimental studies revealed a mismatch of the proposed formulas for description of thermal electron emission. When relatively weak electric fields were superimposed, wave-like oscillations of the emission current were observed near the Schottky line $\left(\lg i=f\left(U_{B}^{1 / 2}\right)\right.$, where ( $U_{B}$ is the inter-electrode potential). As a result, it was suggested that electrons should be reflected from the walls of this barrier when passing through a potential barrier, and, as a result of interference of electron waves, a periodic change in the emission current occurs. As a result of this, the interference component was introduced into formula (6), namely:

$$
J_{e}=A_{R} D T^{2} \exp \left(-A_{B} / k_{b} T\right)
$$

Where $D$ is the reflection factor of electrons at the metal-vacuum interface.

Expression (7) is known as the Richardson-Laue-Dashman equation. A. Modinos, a well-known specialist in the emission properties of substances, comments on the current situation as follows: "It should be noted that electron reflection from the metal-vacuum interface and the phenomena caused by it cannot be accurately analyzed even in approximation of free electrons, since reflection of electrons at the interface is closely related to the crystalline nature of the metal and the band structure of its energy levels in most cases" [16].

For each material, formula (7) may be easily coordinated with the experimental data by picking coefficient $A_{R}, A_{B}$ and $D$. But this is pure empiricism. If the form of the potential barrier is not changed, as it follows from the definition of such barrier, then the wave-like behavior of the emission current must arise with the constant amplitude. Different amplitudes are observed experimentally.

A similar situation takes place in field emission. Quantum mechanics interprets field emission as the result of passaging of free electrons through a potential barrier. Such approach causes determination of the following value of the emission current:

$$
J_{e} \approx E_{\ni}^{2} \exp \left(-\frac{a}{E_{\ni}}\right)
$$

In this formula, constant $a$ depends on the magnitude of the work function.

A nice theory has been developed, but it is not correct in the statement of the problem, since leakage is considered through the potential barrier of free electrons that are absent in the condensed state, as they are in the potential well of a positively charged core of a molecule, cluster, or crystal lattice. Electrons in metals obey the Fermi-Dirac distribution law. Their energy does not increase but decreases relative to the Fermi level. The change in the thermal energy of electrons is insignificant as compared to the Fermi level. 
Therefore, the comparison with the experiment was performed qualitatively, and the Fowler-Nordheim functions related to the value of the decrease in the work function described in the Schottky theory were used for the quantitative description [11]. No physical rationale of the Fowler-Nordheim functions is provided. Therefore, the proposed theory of field emission should be considered as a purely empirical theory.

When emission phenomena were discovered and the appropriate models were developed, knowledge of the physics of this phenomenon was limited by the following:

1. It was not clear, which particles were crystals formed of.

2. It was not clear, what a crystalline surface was.

3. It was not clear, what the role of negative ions in crystals was.

4. It was not clear, how was the crystal surface deformed under the influence of an external electric field.

5. It was not clear, how various emission phenomena arose from the surface of crystals.

6. It was not clear, what particles evaporated from the liquid near the boiling point.

\subsection{Theory of Thermal Electron and Field Emission}

It was shown in [11] that thermal electron and field emission arises as a result of vibration of clusters on the surface layer. With that, field emission is considered to be thermal electron emission with many emission centers, taking into consideration the reduction in the work function due to application of the external electric field and without consideration of the presence of negative ions in the restricted zone. The size of the emission spot in the STM corresponds to the diameter of one atom. Therefore, emission of electrons is performed from the single emission center. The Schottky theory is not applicable for the single emission center, and, taking into consideration the presence of negative ions in the band gap and their population, the resulting density of the emission current based on [11] in its most general form will be:

$$
J_{A T}=\frac{e}{4 r_{k}^{3}} \sqrt{\frac{8 k_{b} T}{m_{c}}} \sum_{i}\left(\frac{E_{i} \pm \sigma E_{\ni}^{2}}{k_{b} T}+1\right) \exp \left(-\frac{E_{i} \pm \sigma E_{\ni}^{2}}{k_{b} T}\right) \exp \left(-\frac{\mu-E_{i}}{k_{b} T}\right) \text { Gretchikhin's Formula }
$$

Where $r_{k}$ is the size of the cluster, molecule or atom; $m_{c}$ is the mass of the cluster, molecule or atom. With $i=0 \quad E_{0}=\mu$, and with other values, it is equal to $E_{i}=E A_{i}$. With the absence of the external electric field $\sigma E^{2}=0$ and then formula (9) determines the thermal electron emission. In turn, it follows from (9) that at $E_{i} \pm \sigma E_{\ni}^{2}=0$ the density of the emission current of field emission reaches its limit value and does not depend on the magnitude of the applied external electric field

$$
J_{A T, \max }=\frac{e}{4 r_{k}^{3}} \sqrt{\frac{8 k_{b} T}{m_{c}}} \exp \left(-\frac{\mu-E A_{i}}{k_{b} T}\right)
$$

The surface atoms of the crystal are involved in the thermal electron and field emission, since they contain an excess negative charge of negative ions. For most metals, it was believed that free electrons at the Fermi level participate in field emission, and these electrons overcome the work function by the tunneling effect. Electrons of negative ions do not have a spin and are free. The work function for many crystals is several electron volts. With such values of the work function, the electric current of the field emission under normal conditions on the basis of (10) is quite small, and, therefore, cannot be measured. To increase the field emission current, crystal samples were made with the area that would have a large number of emission centers.

In STM, the needle is made of tungsten, and the electric current of field emission is formed from one atom as the emission center. With that, the following should be taken into consideration. Tungsten has a relatively high binding energy between interacting particles inside the metal and, therefore, different influences do not significantly change its structure and, accordingly, the location of the built-in dipole electric moments. Like any solid body, a tungsten crystal consists of clusters [7], and its crystal lattice has the BCC structure. Clusters of the BSS structure are tetrahedral prisms with 7 atoms. If the end of the needle is created with the size of one cluster, then the end of such needle should have the size of one atom. That is why tungsten in the STM is used as a needle. The size of the tungsten atom in the crystal is $r_{k}=1.41 \AA$. If the voltage of $\sim 2 \mathrm{~V}$ is applied to such needle, then the electric field strength at the surface of the tungsten cluster reaches $E_{\ni}=U / r_{k} \approx 1.42 \cdot 10^{10} \mathrm{~V} / \mathrm{m}$. At such external electric field intensities, the field emission is quite pronounced. For tungsten, the Young's modulus is equal to $E=4 \cdot 10^{11} \mathrm{~Pa}$, $h \cong r_{k}$ and $\varepsilon_{r} \cong 1.01$ [11]. Based on this data, ponderomotive forces will perform the work of $\mathrm{eV}$. In this case, the electric current density under normal conditions will be

$$
J_{A T, \text { max }}=\frac{e}{4 r_{k}^{3}} \sqrt{\frac{8 k_{b} T}{m_{c}}} \exp \left(-\frac{\mu-E A_{i}}{k_{b} T}\right)=4.13 \cdot 10^{-39} \mathrm{~A} / \mathrm{m}^{2}(11)
$$

i.e., almost equal to zero.

Therefore, field emission from a tungsten needle must not affect the occurrence of electric current between the needle and the surface under study. This is a fundamental condition for the use of the STM. If the voltage of more than $4 \mathrm{~V}$ is applied to the tungsten needle, then the electric current of $1.1 \cdot 10^{-7} \mathrm{~A}$ will occur in the discharge gap, due only to field emission from the tungsten needle. In this case, the electric current of field emission characterizes the behavior of tungsten clusters in the electric field, and not the behavior of the surface under study, which is substantially distorted. 
The distance between the moving needle and the test surface must not be less than the radius of the studied Fermi cluster surface. In the case of the studied silicon surface, its radius of the Fermi cluster surface is $1.18 \cdot 10^{-10} \mathrm{~m}$. The ionization energy of the silicon cluster is $2.69 \mathrm{eV}$ [13]. On the Fermi silicon cluster surface, the electric field without taking into consideration the effects of ponderomotive forces is

$$
E_{\ni, S i}=\frac{U r_{0}}{\left(r_{0}+h\right)^{2}}=4.2 \cdot 10^{9} \mathrm{~V} / \mathrm{m},
$$

where $r_{0}$ and $h$ are, correspondingly, the radius of the tungsten needle and the radius of the silicon cluster. The time of passage of the discharge gap by the electron is $\sim 3 \cdot 10^{-12} \mathrm{~s}$. In this case, the electric current is equal to $\sim 5.3 \cdot 10^{-8} \mathrm{~A}$. If the time of variation of the voltage fed to the needle or the time of the period of oscillation of the silicon cluster significantly exceeds the time of closure of the discharge gap, this will not change the result of measurement of the changing discharge current in the gap between the tungsten needle and the plane of the silicon surface under study.

Summarizing the theory of the thermal electron and field emission, the following should be noted:

1) The emission current of electrons of the thermal electron and field emission is determined by the probability of population of the energy levels of negative ions in the restricted zone, as well as the effects of temperature fluctuations of surface clusters and the external electric field.

2) The field emission occurs sequentially until the applied voltage to the discharge gap, multiplied by the electron charge, becomes equal to the electron affinity for the emission negative ion.

3) The emission current reaches its limit value and remains constant with the increasing intensity of the external electric field.

4) With the positive voltage, the electric current of field emission significantly exceeds the current that occurs with the negative voltage supplied to the cantilever needle.

These theoretical conclusions must be experimentally proved.

\section{Experimental Studies}

The dependence of the emission current magnitude on the applied voltage and its sign at different points on the surface was studied by different authors $[17,18]$. As a result, data was obtained that is difficult to interpret, as monitoring of the conditions for applying the STM was not sufficiently controlled. Therefore, under strict control of the STM application conditions, electric currents of field emission in two halves of rhombus $\mathrm{FH}$ and $\mathrm{UH}$ were obtained on the surface $^{1}$ of the silicon crystal. See the result in Figure 8:

1 Consideration of the two halves of the isolated rhombus is due to the fact that when indium sputtering a silicon surface of $\mathrm{Si}(111)$ at first one half of $\mathrm{UH}$ is completely filled, and then $\mathrm{FH}$, and this is due to the different binding energy of

\section{di/dV, nAN}

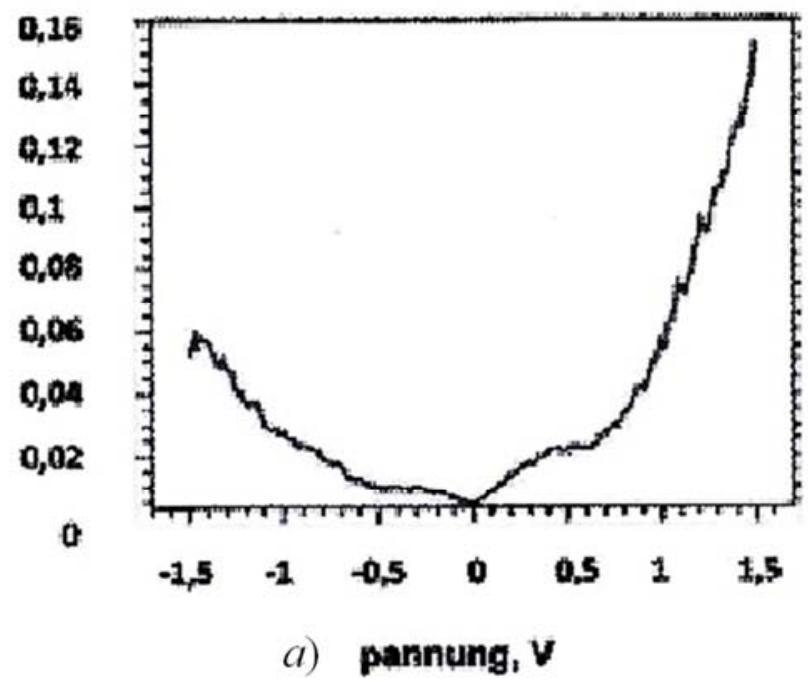

do/du,naps

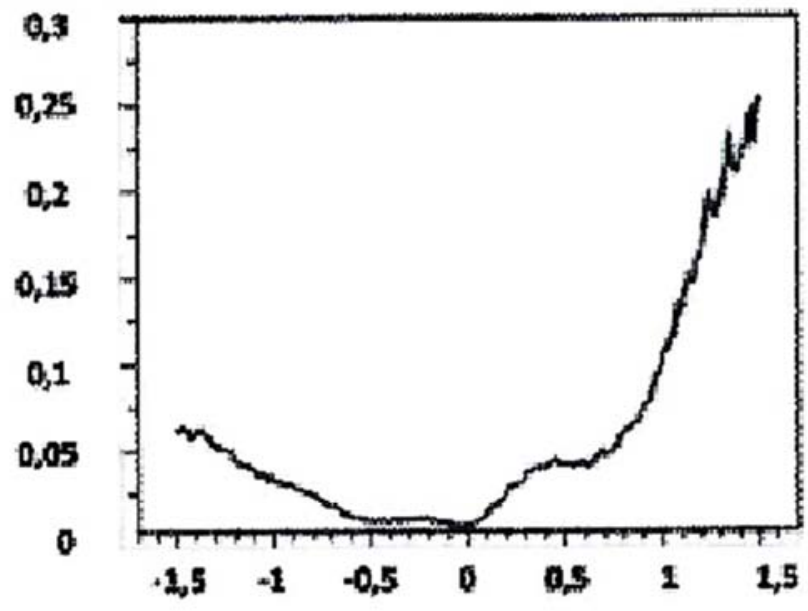

b) spannuang. $\mathbf{v}$

Figure 8. The dependence of the electric current of field emission for a silicon crystal obtained in different places of the Si (111) rhombus surface: a) the UH half of the rhombus and b) the FH half of the rhombus.

It turns out that the emission properties of silicon molecules located on different halves of the rhombus are not significantly different. This is natural, since the field emission occurs from the same molecule.

In the case of silicon, field emission starts at $0.03 \mathrm{~V}$ and reaches the maximum at the distance of 0.4-0.5 V. From the value of $0.5 \mathrm{~V}$, a sharp rise occurs, and from the value of 1.4-1.5 V, the increase in the emission current is so significant that it was necessary to stop performing measurements.

Electrons in negative ions interact with the built-in dipole electric moment. They have no spin and are free to participate in the field emission along with the electrons located at the Fermi level. Therefore, electrons at the Fermi level and at the energy levels of negative ions participate in the field emission.

indium clusters on the silicon surface. 
The field emission begins when $e U=\left|E A_{i}\right|$. The stepwise increase in the field emission current exactly corresponds to all three values of electron affinity for the basic state of silicon, which is also confirmed by the measurements made in $[12,14]$. The obtained experimental data convincingly indicate that the electric current of the field emission is formed by the electron emission mainly from negative ions.

To confirm this conclusion, we measured the field emission current for indium, the atoms of which have the electron affinity of $0.3 \mathrm{eV}$. Indium in the crucible was heated to the boiling point, and flat indium clusters of three diatomic molecules were deposited on the silicon surface [14]. See the result of measurements of the field emission current in Figure 9.

\section{did' n:SW}

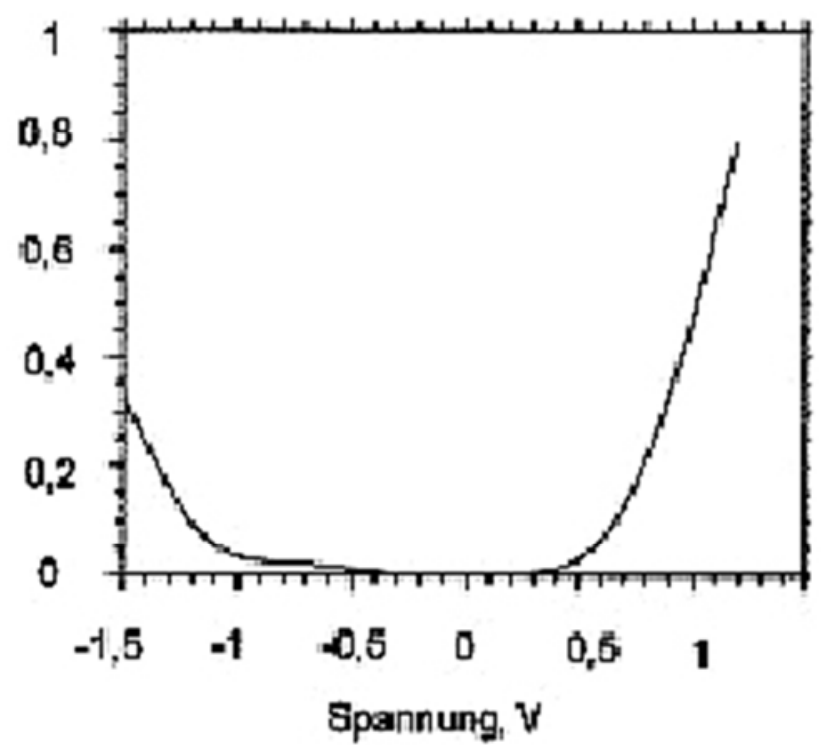

Figure 9. Dependence of the field emission current for indium depending on the voltage applied to the cantilever.

The field emission for indium begins with the voltage of 0.3 $\mathrm{V}$ on a tungsten needle and is increased exponentially in accordance with formula (9).

\section{Conclusions}

The theoretical and experimental studies performed indicate the following:

1) The conditions of applicability of a scanning tunneling microscope for the analysis of the emission portrait of the crystalline surface studied are established.

2) It was shown that the thermal electron and field emission of electrons in a scanning tunneling microscope is implemented from a single emission center.

3) Ponderomotive forces in a scanning tunneling microscope significantly distort the structure of the crystal surface studied, which causes a noticeable decrease in the field emission current at the negative voltage as compared to the positive voltage on the STM cantilever.

4) It was experimentally established that triatomic molecules possess allotropic arrangement of internal dipole electric moments of compound atoms.

5) The theory of field emission from a single emission center was developed.

6) It was theoretically and experimentally established that the field emission in a scanning tunneling microscope is performed jointly from negative ions and the Fermi level.

7) The electric current of field emission begins with the magnitude of the voltage applied corresponding to the value of the affinity energy of the surface layer atoms.

8) The field emission current for each negative ion is saturated.

9) Noticeable influence of the electric field strength square on the auto-electronic emission occurs when $\left(E_{i} \pm \sigma E^{2}\right) \triangleright k_{b} T$.

As a result, it was theoretically and experimentally proved that all previously developed models of the thermal electron and field emission do not correspond to the real physics of the phenomenon. Thermoelectronic emission arises due to the temperature population of negative ions and the fluctuation of the surface layer of clusters in the crystal. Field emission is thermal electron emission from negative ions and the Fermi level, which is transformed due to the action of the external electric field on the work function and deformation of the surface with ponderomotive forces.

\section{References}

[1] Gretchikhin L., Komarovskaya V. Negative ions of atoms, diatomic and triatomic molecules. // Military technical Courier. Scientific Periodical of the Ministry of Defence of the Republic of Serbia. 2016. 64 (2), 447- 464.

[2] Gretchikhin L. I. Solids interaction with environment in near-Earth space. (Gretchikhin effect). // Materials of the First Belarusian Space Congress on October 28-30, 2003 in Minsk: UIIP NAS of Belarus, 2003-31-33.

[3] Gretchikhin L. Negative ions in space exploration. / VIII World congress "Aviation in the 21st century"-"Safety in Aviation and Space Technology" 10-12 october 2018 of the year. Kiev. NAU P. 2.3.29-.3.37.

[4] Gretchikhin L. I. Formation of p-, conductivity in semiconductors /Military technical Courier. Scientific Periodical of the Ministry of Defence of the Republic of Serbia. 2018. V. 66, № 2, P. 304-321.

[5] Gretchikhin L. I. Formation of p-, n-conductivity and p-n junction. /Reinforcing technologies and coatings. 2018. № 5. P. 57-63.

[6] Gretchikhin L. I., Latushkina S. D., Komarovskaya V. M. Doube electrical layer in cathode spot. / Military technical Courier. Scientific Periodical of the Ministry of Defence of the Republic of Serbia. 2016. 64), № 3, P. 670- 689.

[7] Gretchikhin L. I. Physics of Nanoparticles and Nanotechnologies. General bases, mechanical, thermal and emission properties. - Minsk: UP "Technoprint". 2003. 399 p.

[8] Gretchikhin L. I. Nanoparticles and nanotechnologies.-Minsk: FLLC "Law and economics".) 2008. 406 p. 
[9] Landolt-Börnstein/Zählenwerte und Funktionen, 6 Auflage, Springer-Verlag, 1957. IV Bd, 3 Teil.

[10] Makogonyuk G. D., Aidarov V. M., Sedov A. D. Nanostructure of the surface of crystals obtained from solutions subjected to various physical effects / Ulyanovsk Technology Transfer Center: Collection of project summaries of the Youth Innovation Forum of the Volga Federal District (Ulyanovsk State Technical University, May 12-14, 2009).-Ulyanovsk: Ulyanovsk State Technical University, 2009. P. 183.

[11] Gretchikhin L. I. Physics. Electricity and magnetism. Modern electrodynamics. Minsk: FLLC "Law and Economics". 2008. $302 \mathrm{p}$.

[12] Gretchikhin L. I., Latushkina S. D., Komarovskaya V. M., Shmermbekk Yu. Cluster structure of silicon and its surface structure.//Reinforcing technologies and coatings. 2015. № 9. P. $9-16$.

[13] Physical quantities. Handbook./A. P. Babichev, N. F. Babushkina, A. M. Bratkovsky, etc.; Edited by I. S. Grigorieva, E. Z. Meylikhova.-M.: Energoatomizdat, 1991,-1232 p.

[14] Gretchikhin L. I., Latushkina S. D., Komarovskaya V. M., Shmermbekk Yu. Formation of densely packed and cluster lattice structure of indium on the surface of silicon./Magazine "Strengthening Technologies and Coatings" № 6, 2015. P. 3-12

[15] . Sivukhin D. B. General physics course. Mechanics.-M.: "Science" 1979-519 p.

[16] Modinos A. Auto-, thermo- and secondary electronic emission spectroscopy: /Edited by G. N. Fursei. M.: Science, 1990, -320 p.

[17] Wolkov R. And Avouris Ph. Atom-Resolved Surface Chemistry Using Scanning Tunneling Microscopy//Phys. Rev. Let. 1988 Vol. 60 № 1

[18] Li J. L., Jia J. F., Liang X., J. Et al.//Phys Rev. Let. 2002. Vol. 88. P. 066101 (1-4).

[19] Gretchikhin L. I. Densely packed state is the basis of nanotechnologies. /Mechanical Engineering and Technosphere of the XXI Century // Proceedings of the XIX International Scientific and Technical Conference in Sevastopol, September 17-22, 2012. In 3 volumes.-Donetsk: DonNTU, 2012. T. 1. P. 195-197. 